System Dynamics Review

System Dynamics Review vol 28, No 1 (January-March 2012): 46-68

Published online 24 January 2012 in Wiley Online Library

(wileyonlinelibrary.com) DOI: 10.1002/sdr.476

\title{
Mental models of dynamic systems: taking stock and looking ahead
}

\author{
Stefan N. Groesser ${ }^{\mathrm{a}, \mathrm{b} *}$ and Martin Schaffernicht ${ }^{\mathrm{c}}$
}

\begin{abstract}
This paper deals with the representation of the conceptual structure of mental models of dynamic systems (MMDS). Heretofore, this structure has not been consistently defined. Consequently, studies about MMDS continue to use different conceptual structures to measure mental models. Even such properties as feedback loops and delays, which lie at the core of dynamic systems, are often not considered. This situation leads to incompatible findings and stagnating research. We review the literature about mental models in the field of system dynamics. In addition, we refer to dynamic systems theory as the mathematical basis for system dynamics to complement and validate our conceptual structure. One may conclude that most of the existing mental model studies measure only parts of the structure that we propose. The paper's contribution is to elaborate the conceptual structure of an MMDS and to use this structure to operationally enhance the definition of an MMDS. Copyright () 2012 System Dynamics Society.
\end{abstract}

Syst. Dyn. Rev. 28, 46-68 (2012)

Additional supporting information may be found in the online version of this article.

\section{Introduction}

Extensive research has shown that humans perform poorly in coping with dynamically complex systems (Sterman, 1989a, b; Moxnes, 2000, 2004; Sterman and Sweeney, 2002; Dörner, 2006; Sweeney and Sterman, 2007). The first aspect of the problem is the inability of humans to mentally infer the dynamic behavior of accumulation processes-the so-called "stock and flow failure" (Cronin and Gonzalez, 2007; Cronin et al., 2009; Brunstein et al., 2010; Sterman, 2010). Second, humans also fail to recognize causal feedback relations that are distant in time and space (Moxnes, 2004; Sterman, 2008). To comprehend the behavior of dynamically complex systems, a mental model is required that accounts for accumulation processes, time delays, and feedback loops.

For understanding in detail why humans are incapable of accounting for dynamics, it is highly relevant to conceptualize the content of the mental models humans use to make decisions. Mental models are abstract representations of situations which individuals maintain in their minds (Forrester, 1961). More formally, "mental models reflect the beliefs, values, and assumptions that we personally hold, and they underlie our reasons for doing things the way we do" (Maani and Cavana, 2007, p. 15); Argyris (1982) calls them "theories-in-use"; for Senge (1990) they are "internal images of how the world works" (p. 174). Research into mental models tries to explore, among other topics, the structure

\footnotetext{
${ }^{a}$ System Dynamics Group, Institute of Management, University of St Gallen, Dufourstrasse 40a9000, St Gallen, Switzerland.

${ }^{\mathrm{b}}$ Bern University of Applied Sciences, Department of Business, Morgartenstrasse 2c, 3005 Bern, Switzerland.

${ }^{\mathrm{c}}$ Universidad de Talca, Facultad de Ciencias Empresariales, Avenida Lircay s/n3460000, Talca, Chile.

* Correspondence to: Stefan N. Groesser. E-mail: stefan.groesser@unisg.ch

Received 15 December 2010; Accepted 16 September 2011
}

Copyright @ 2012 System Dynamics Society 
of mental models, changes of mental models over time, and the determinants of these changes (Walsh, 1995; Schaffernicht, 2006; Hodgkinson and Healey, 2008).

Doyle and Ford $(1998,1999)$ have introduced the notion of a mental model of a dynamic system (MMDS). This type of mental model captures dynamic aspects of reality. In the words of Doyle and Ford, an MMDS is "a relatively enduring and accessible, but limited, internal conceptual representation of an external system whose structure is analogous to the perceived structure of that system" (Doyle and Ford, 1999, p. 414). However, the phrase "whose structure is analogous to the perceived structure of that system" is unclear. The following important questions are left unanswered: What is the structure of a dynamic system? What are its building blocks? How can these building blocks be represented? These questions describe the gap that we address.

The paper contributes to substantiating the unspecified term "structure" of the definition by Doyle and Ford $(1998,1999)$. It elaborates the building blocks of dynamic systems and thereby details the conceptual structure of an MMDS which consists of variables, causal links with their polarities and significant delays, as well as feedback loops, their polarity, and their nonlinearities. Before doing this, however, we should be clear about the scope of the paper. The intent is not to discuss aspects of mental models of static systems as they are used in other fields of research, e.g. psychology or organizational research. Here, we concentrate on explicating the structure of an MMDS by means of reviewing existing MMDS studies and constructing an analogy to dynamic systems theory.

To achieve this explicit representation of the system's dynamic structure is of high relevance because it has potential for significantly advancing the field of MMDS research. A more accessible conceptual structure would ease future research into measuring and comparing mental models; for instance, measuring and comparing the effects of interventions on learning. Only recently, interest within the system dynamics community in mental model research has increased (Kunc and Morecroft, 2010; Plate, 2010; Sterman, 2010). However, all available studies use different operationalizations of an MMDS and therefore cannot be compared with each other. Hence, with a conceptual structure that is widely available, researchers could better build on each other's work. Schaffernicht and Groesser (2009, 2011) provide a comparison method which allows researchers to benefit more from work by others, thereby accelerating research into MMDS using system dynamics. One can foresee that the need for research into dynamic decision making, e.g. in strategy research (Gary et al., 2008; Gary and Wood, 2011), could be reliably addressed with this advance in accessibility.

The article is structured as follows: the next section briefly introduces the concepts of a mental model and an MMDS and shows the difference between them. Thereafter, we review research into MMDS and develop a preliminary conceptual template for use in this research. However, since this conceptual structure lacks important elements, in the fourth section we introduce dynamic systems theory, which is also the mathematical basis of system dynamics. It provides us with a robust foundation of elements used for presenting an MMDS. In the fifth section, we review the structural elements of system dynamics in order to relate them to the elements of dynamic systems theory. This synthesis is carried out in the sixth section. Based on the best available sources (existing research, dynamic systems theory, and system dynamics) to achieve our objective, we derive a comprehensive and parsimonious conceptual structure for MMDS. This structure is also used to operationally enhance the definition of MMDS. Before the conclusions, the sixth section points also to limitations of the structure we propose in this paper. 


\section{The mental model concept}

\section{Mental models}

The mental model concept is not a recent invention. It can be traced back to Kenneth Craik (1943), who proposed that thinking is the manipulation of internal representations of the world. In the field of psychology, Johnson-Laird (1983) has elaborated a theory of human reasoning which uses mental models. It deals with how humans resolve problems using mental representations of what they believe to be true (Johnson-Laird, 2001). Mental models as the basis for a theory of reasoning are an intensively researched topic in psychology. According to the current understanding of mental models, each known fact or relationship between facts is represented as a logical assertion (Seel, 2001). The following example demonstrates how assertions are used to infer consequences: "Dry conditions and a burning cigarette can cause a forest fire", "Today the weather is dry", and "Someone throws away a burning cigarette"; the result is that a fire can be caused. When two or more such assertions are linked together, they constitute a mental model. When there is more than one reason for an outcome to occur, it is possible that several mental models exist in parallel to explain that outcome (Goldvarg and Johnson-Laird, 2001; Seel, 2001). These mental models are then used to evaluate the possibilities and consequences and to arrive at a conclusion that is valid for the circumstances considered (Johnson-Laird, 1999). Doyle (1997) discusses additional conceptual knowledge representations in psychological and cognitive research, e.g. "schemas" and "scripts", and relates them to the field of system dynamics.

\section{Mental models of dynamic systems}

The treatment of mental models in psychological research is not useful for understanding mental models of dynamic systems as defined by Doyle and Ford $(1998,1999)$ for several reasons.

First, the problem situations which confront the subjects in traditional mental model research often have static, open-loop characteristics and usually involve actions and events rather than continuous and closed-loop processes. Hence the treatment of mental models as linked sets of assertions is not helpful for understanding dynamic phenomena.

Second, logical assertions imply system structure, but they do not separate it from system behavior. It follows that if the underlying causal structure allows different system behaviors to unfold, there will be as many mental models as there are possible behaviors. This way of representing structure is inefficient since the structure is not disentangled from behavior. Also, the structural part is represented multiple times. The representation of mental models of dynamic systems is more efficient. The focus is on the structural part of the mental model, i.e. variables and causal links, but not the behaviors that can emerge from the structure. For instance, in any given assertion, e.g. "it is hot", a variable is implicitly used; in our case, "temperature". Other aspects of the assertion "is hot" are not used since it is not a causal statement. An MMDS, therefore, is a model of the underlying system structure that contains the relevant mechanisms to explain the emergence of specific situations.

A third difference between traditional mental model research and research into mental models of dynamic systems (Forrester, 1968b; Eden, 1988, 1992, 1994; Markóczy and 
Goldberg., 1995; Lane, 1999; Doyle et al., 2002, 2008; Rosenhead and Mingers, 2001) is that the latter is specifically concerned about how and why the mental models of subjects change over time, and not how they are used to reason about a given task. Arguably, the cognitive mapping approach (Bryson et al., 2004; Ackermann and Eden, 2011) is close to MMDS. It deals with dynamic situations, uses diagramming techniques to structure problems, and strives to articulate the causal understanding of problem owners. However, there are also differences. Because cognitive mapping is based on construct theory, the intention is to elicit constructs and not variables; in addition, causal structure is not separated from its behavior, which is one principle of system dynamics. Finally, the elements of a conceptual structure in an MMDS cannot be expressed by the elements used in cognitive mapping diagrams. These differences, at the same time, however, make cognitive mapping a fruitful complementary method for system dynamics studies (e.g. Howick et al., 2006).

Even though the notion of an MMDS (Doyle et al., 1998; Doyle and Ford, 1999) marks a significant advance, it falls short of providing an explanation of the properties of a mental model's structure. Doyle and Ford (1998) themselves assert that the precise nature of this structure has yet to be elaborated. Also, Senge (1990) mentions MMDSs which are "mental models that recognize longer-term patterns of change and the underlying structure producing those patterns" (p. 204). But again concretization of the structure is missing. We will conduct a review of the relevant literature to understand whether the definition of an MMDS has been used or substantiated.

\section{Research into mental models of dynamic systems}

For the review, we have selected studies that try to measure mental models of dynamic systems. We accessed Thomson Reuters's Web of Science database and employed searches using both isolated and combined strings of the following terms: "mental model", "dynamic system", "measuring", "structure" and reasonable variations thereof. We also accessed conference proceedings (Winter Simulation Conference, Operational Research Society UK, European Operational Research Conference, System Dynamics Society). We focused especially on the streams of dynamic decision making and natural decision making. We can conclude that MMDSs have been addressed especially in the field of system dynamics. Since the object of mental model research in psychology focuses on events and static situations, we include psychological studies in our review only when they add to the conceptual structure of MMDS. As our review shows, the following elements to operationalize the structural representation of MMDS have been measured: causal links, link strength, link polarities, variables, feedback loops, and, less often, other properties such as length of a feedback loop (Table 1).

\section{Causal links}

Most of the studies we have reviewed examine causal links as the fundamental element of a mental model. Some studies compare several explicated mental models and try to find common causal links. The first two studies of Table 1 (Ritchie-Dunham, 2002; Capelo 
50 System Dynamics Review

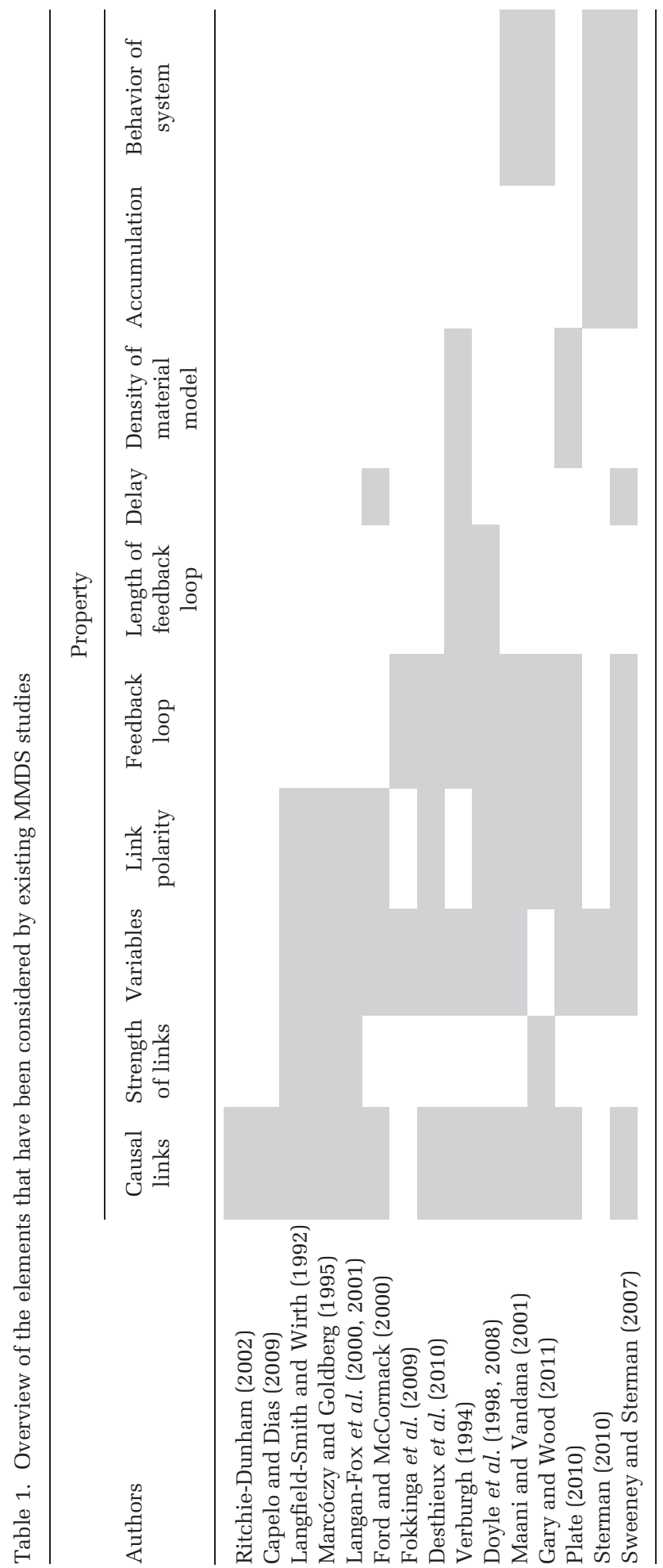


and Dias, 2009) account only for causal links. The method which compares causal links is described in Schvaneveldt (1990).

\section{Variables, link strength, and link polarity}

In addition to causal links, the studies by Langfield-Smith and Wirth (1992), Markóczy and Goldberg (1995), and Langan-Fox et al. $(2000,2001)$ consider also the polarity of the causal links, the strength of the causal link, and the associated variables. Variables represent nodes in the structure of a mental model where the links begin and where they end. The links represent cause-effect connections between two variables with either a positive or a negative direction of causality. In addition, causal relations are specified by their strengths.

The conceptual elements of an MMDS which we have reviewed up to now are causal links, link polarity, link strength, and variables. The concept of "variables" has been used as a general category; no fine-grained differentiation has been employed. Moreover, if the conceptual elements that have been studied so far (Table 1) are not interrelated in a specific manner, then these elements cannot endogenously explain dynamic situations, i.e. the development of a quantity over time (Senge, 1990). But what property is it that enables a (mental) model to endogenously represent a dynamic system?

\section{Feedback loops}

Feedback loops enable a model system to endogenously represent dynamic aspects of a system (Forrester, 1968b; Richardson, 1999). A feedback loop is a logically closed causal chain where an initial change in a variable is fed back to its origin. The reviewed studies about MMDS compare the feedback loops in the mental models of different persons (Verburgh, 1994; Fokkinga et al., 2009; Desthieux et al., 2010; Plate, 2010), within the same person (Doyle et al., 1998, 2008; Sweeney and Sterman, 2007), or between persons and an optimal system structure represented in a computer system (Ford and McCormack, 2000; Gary and Wood, 2011). Doyle et al. $(1998,2008)$ account for all other elements, besides feedback loops, that we have discussed until now. Others use only some elements in addition to feedback loops. For instance, Fokkinga et al. (2009) consider variables, but do not account for links, link strength, and link polarities; Gary and Wood (2011) account for causal links, strength of the links, and link polarity. Ford and McCormack (2000) concentrate on delays in links.

\section{Other properties}

In addition to the properties we have just reviewed, researchers have measured other aspects of mental models. Table 1 shows that most of these properties are used only in a few studies, often only one. Verburgh (1994) and Sweeney and Sterman (2007) have measured indications of delays, an aspect which has not been explicitly measured by others. Verburgh, in addition, computes the length of feedback loops, measured in number of variables involved in the loop, and the density of the mental model computed as a ratio of links of the model in relation to the maximum possible number of links. This density is also considered by Plate (2010). The issues introduced by Verburgh have been elaborated 
by Doyle et al. (2008). They compute a normalized maximal path length and a normalized length of a feedback loop.

An additional aspect of dynamic systems is considered by Gary and Wood (2011), who measured the mental model owners' expected behavior of the system. The authors showed five feasible behavior-over-time graphs for a variable. The participants had to select the curve which they expected to be most appropriate. Also Sterman (2010) and Sweeney and Sterman (2007) have measured the participants' expectation of a variable's behavior under different scenarios. In addition, Sterman and Sweeney measure the topic of accumulation. Maani and Vandana (2001) measure actual or expected behavior that participants have verbalized during a task.

We can see that there are ten properties of MMDS that researchers have measured (Table 1). The reviewed studies use on average less than half of the properties of an MMDS; only four studies use more than five of the ten properties. The properties which are most often considered are causal links, variables, link polarity, and feedback loops. It is rather astonishing that feedback loops, the core of a dynamic system, are accounted for in only half of the studies. In addition, the concept of delays and accumulations has been addressed only rarely. We have condensed the properties of Table 1 in an abstracted set of concepts (Figure 1).

Considering the variety of elements and their inconsistent use in the studies, it is not ascertainable which elements form a conceptual structure of MMDS. No consolidated body of research exists that would allow one to reach such a conclusion. This is why we turn now to dynamic systems theory. It can support us in validating and complementing the list of components to form a sufficient and parsimonious conceptual structure for MMDS. With the selection of the dynamic systems theory, we follow the path already demonstrated by Richardson (1999), who has argued that any dynamic system can be represented as a feedback system using the system dynamics notation. We detail his argumentation on the level of elements.

\section{Dynamic systems}

Put simply, "a dynamic system is one whose state changes with time $t$ " (Arrowsmith and Place, 1990, p. 1), or a dynamic system is a means of describing how one state develops into another state over the course of time (Wolfram, 2002; Arnold, 2006). In dynamic systems theory, discrete dynamic systems and continuous dynamic systems are differentiated.

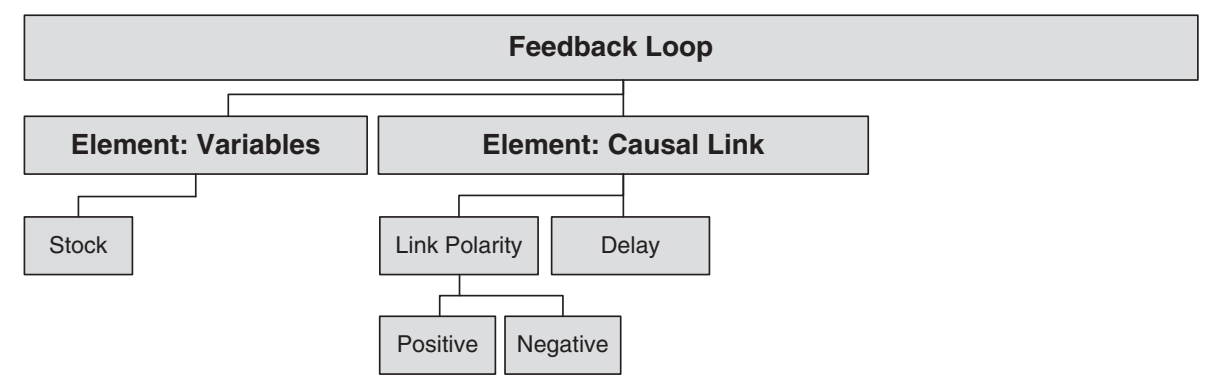

Figure 1. Summarized conceptual structure currently used in mental model studies 
For our purposes, we refer only to the latter type. The dynamics are usually described by a differential equation in the form of $\frac{\mathrm{d} x}{\mathrm{~d} t}=\dot{x}=f\left(x_{t}, t\right), x_{t}$ being the state of the system at time $t$ and $\dot{x}$ being the change of this state. The theory of dynamic systems is mature and has been beneficially used in social sciences to describe dynamic phenomena, e.g. in psychology (Jensen and Brehmer, 2003; Smith and Thelen, 2003), strategic and organizational management (Sterman et al., 1997, 2007; Anderson, 1999; McKelvey, 1999; Fowler, 2003; Amaral and Uzzi, 2007; Warren, 2008), political science and sociology (Miller and Stadler, 1998), chaos research (Brown, 1995; Hufford et al., 2003), economics (Billard, 1977; Kozyk et al., 2010), and environmental research (May and Oster, 1976). Because of this wide applicability, the theory provides a profound basis from which to depart in order to validate and complement the components of a conceptual structure of MMDS.

\section{Example of a dynamic system}

We use the Lotka-Volterra (LV) predator-prey model (see supporting information; Lotka, 1910; Volterra, 1926; Goodwin, 1967; Desai and Ormerod, 1998) to introduce the essential elements of a second-order, nonlinear dynamic system. We use this model because, first, it is the dynamic model which most social scientists know immediately, and second, it is complex, but also easy to comprehend. It depicts the interaction of two populations which mutually influence each other, resulting in sustained oscillatory behavior. The prey population at time $t, \operatorname{Prey}_{t}$, is a state variable of the system which is determined by its initial value, $\operatorname{Prey} P_{\mathrm{t}=0}$, and by the related transition functions birth of new prey, $b P r e y_{t}$, and death of the prey, $\operatorname{dPrey}_{t}$ (Eq. 1):

$$
\begin{gathered}
\text { PreyP }_{t}=\int_{0}^{t}\left(\text { bPrey }_{t}-d \text { Prey }_{t}\right) \times \mathrm{d} t+\text { PreyP }_{0} \\
\text { bPrey }_{t}=\alpha \times \text { Prey }_{t-1}
\end{gathered}
$$

The transition functions are determined by additional factors. For the case of prey births, its amount at any given time depends on the existing population of prey, Prey $P_{\mathrm{t}-1}$, and the normal prey birth rate, $\alpha$ (Eq. 2). The number of prey deaths at any given time assumes the maximum amount of the components given in Eq. 3. The average expected life time of the prey, $\beta$, determines the average deaths among the prey population, PreyP $P_{\mathrm{t}-1}$. The crowdedness of prey, PreyCrow $w_{t}$ (Eq. 4), given limited resources, represented by carrying capacity, $C C$, moderates the average deaths according to the nonlinear crowding-death relationship, $\Omega$ :

$$
\begin{gathered}
\text { PPrey }_{t}=\max \left[\frac{\text { PreyP }_{t-1}}{\beta} \times \Omega\left(\text { PreyCrow }_{t-1}\right), \text { Pred }_{t-1}\right] \\
\text { PreyCrow }_{t}=\frac{\text { Prey }_{t}}{C C}
\end{gathered}
$$

The second component of prey deaths is given by the consumption of prey by predators, Pred $C_{t-1}$. This consumption (Eq. 5) depends on the predator population, PredP $P_{t}$, the 
predator food requirements, $\gamma$, and the effect of prey crowdedness, PreyCrow $w_{t}$, on consumption represented by the nonlinear relationship "predator prey consumption" or $\Delta$ :

$$
\text { Pred }_{t}=\gamma \times \text { PredP }_{t} \times \Delta\left(\text { PreyCrow }_{t}\right)
$$

The predator population, $P r e d P_{t}$, is determined by the transition functions, $b P r e d_{t}$, $d$ Pred $_{t}$, and the initial predator population, PredP $P_{t=0}$ (Eq. 6).

$$
\begin{gathered}
\text { PredP }_{t}=\int_{0}^{t}\left(\text { bPred }_{t}-d \text { Pred }_{t}\right) \times \mathrm{d} t+\text { PredP }_{0} \\
\text { bPred }_{t}=\delta \times \text { PredP }_{t-1}
\end{gathered}
$$

The births of predators depend directly on the predator population, $\operatorname{Pred} P_{\mathrm{t}-1}$, and the normal predator birth rate, $\delta$ (Eq. 7). The deaths of predators, $\operatorname{dPred}_{t}$, depend on the average number of deaths, which is moderated by a food-mortality function, $\Phi$ (Eq. 8). The average number of dead predators per given time is calculated on the average life time of a predator, $\lambda$, and the predator population, $\operatorname{PredP}_{\mathrm{t}-1}$. The moderation effect of the nonlinear food-mortality function accounts for the effect which availability of food for predators, PredFA $A_{t-1}$, has on their mortality. Food availability depends on the predator population, PredP $P_{t}$, the predator consumption of prey, Pred $C_{t}$, and the predator food requirements, $\gamma$ (Eq. 9):

$$
\begin{gathered}
\text { Pred }_{t}=\frac{\text { PredP }_{t-1}}{\lambda} \times \Phi\left(\text { Pred FA } A_{t-1}\right) \\
\text { PredFA }_{t}=\frac{\text { Pred } C_{t}}{\gamma \times \text { PredP }_{t}}
\end{gathered}
$$

\section{Fundamental components for representing dynamic systems}

Based on our example, we can flesh out fundamental components which are required to represent dynamic systems. These are:

- State variable. A state variable is one that describes the current condition of a dynamic system. In the LV model, for example, PreyP and PredP denote state variables. At least one variable in the model of a dynamic system must represent a system state which then is changed over time by means of transition functions; this is the only way that a state variable can change (Ogata, 1998).

- Transition function. A transition function describes how a system's state changes over time. It depends on a combination of endogenous variables and exogenous parameters; bPrey and dPrey are examples in the LV model. In general, at least 
one state variable is required for the formulation of a transition function (Arrowsmith and Place, 1990).

- Causal relationship and its polarity. Causal relationships connect variables; the presence of arithmetic operators implies the attribution of causal relationships between the connected variables. If a variable is used in the formulation of an equation, it has a causal relation with a sense of polarity. This polarity can be either positive (all else equal, an increase in variable $X$ increases variable $Y$ above what it would have been) or negative (all else equal, an increase in variable $X$ decreases variable $Y$ below what it would have been). For instance, bPred increases when the PredP increases (given that other conditions remain equal). PredP has therefore a positive relation to $b P r e d$.

- Nonlinear relationships. A system exhibits nonlinear relationships when changes in the output are not proportional to changes in the input. More technically, the dependent variable of a nonlinear relationship cannot be written as a linear combination of independent components. Nonlinearity often indicates that there are interaction effects between variables. Nonlinearity is difficult to analyze intuitively and mathematically, especially when embedded in closed causal relationships (Barlas, 1989; Ogata, 1998). The LV model has three such relationships, e.g. the effect of PredFA on dPred.

- Circularity. Circularity refers to a closed chain of causal relations. This is indicated in differential equations when, for instance, the prey population, $\operatorname{Prey} P_{t}$, depends on its own previous value, $\operatorname{Prey} P_{t-1}$.

- Delay. In dynamic systems, cause and effect are separated in time. The integration processes which take place with respect to state variables lead to such a separation between cause and effect, i.e. a delay between cause and effect. In other words, a delay occurs "if its [the system's] present output depends on past input" (Ogata, 1998, p. 2).

By means of the LV model as an example, this section has elaborated the relevant components of the dynamic systems theory which are widely used to represent the characteristics of dynamic systems. In the next section, we represent the same LV model by means of the methods used in system dynamics. In the sixth section, we bring both approaches together and demonstrate to what degree the elements of a dynamic system can be represented in system dynamics. This matching also provides us with elements of the conceptual structure of MMDS.

\section{System dynamics representation}

System dynamics posits that a system's behavior emerges from its underlying causal feedback structure (Forrester, 1961; Richardson, 1999). Causal loop diagrams (CLD) and stock-and-flow diagrams (SFD) are used to represent this causal structure. The SFD of the LV model is shown in Figure 2. The equations in the diagram are numbered from 1 to 9; the numbers refer to the equations introduced above.

The populations of prey and predators, PreyP and PredP, are represented as stock variables, and the respective birth and death rates, bPrey, $d$ Prey, bPred, dPred, as flow variables. The initial values of the stocks, $\operatorname{Prey} P_{0}$ and $P r e d P_{0}$, the growth and birth parameters, $\alpha, \beta$, $\gamma, \delta$, and the predators' food requirements, $\gamma$, are exogenous parameters. The nonlinear effects of prey crowdedness on predator's consumption, $\Delta$, the crowding effect on prey deaths, $\Omega$, and the predator mortality function, $\Phi$, are included as nonlinear table functions. 


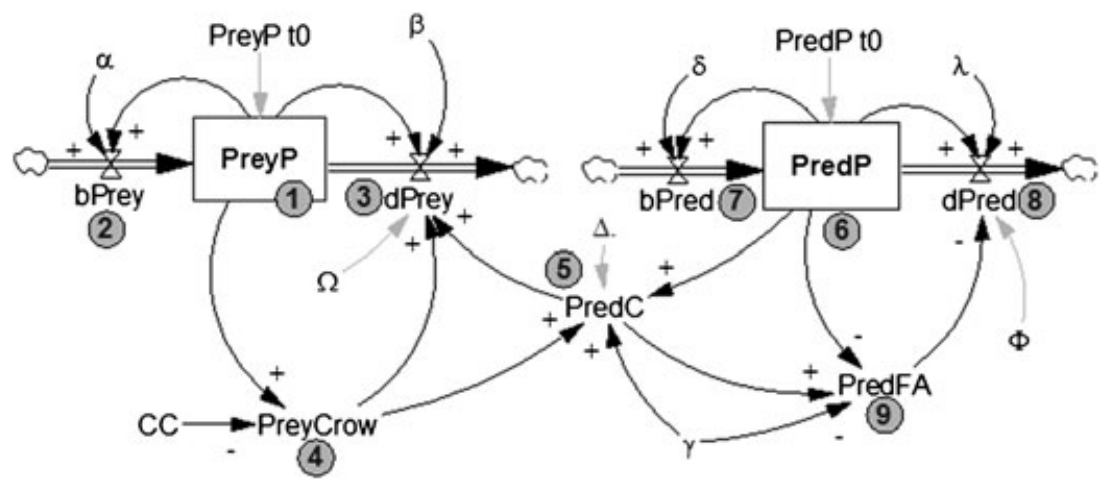

Figure 2. Stock and flow representation of the LV model

The system dynamics representation explicates the causal relationships between the variables as causal links, each of which possesses a positive or negative polarity. The interest of the system dynamicist lies also in the variables: crowdedness of prey, PreyCrow; predator consumption, PredC; and food availability for predators, PredFA. These intermediate variables are used to explain the flow rate equations and thereby explicate the causal structure. The SFD (Figure 2) explicitly represents stocks, flows, and auxiliaries, and it shows all relevant causal relationships. The system structure can also be redrawn as a casual loop diagram (CLD) (Figure 3).

The CLD represents the model in a way that emphasizes feedback loops and delays (Richardson, 1999; Sterman, 2000; Lane, 2008). Feedback loops are indicated by a loop signifier ( $\mathrm{R}$ or B), while delays are represented by a double line orthogonal to a causal link (e.g. between PreyCrow and dPrey). A CLD does not differentiate the type of variables in stocks, flows, and intermediate variables. To combine the strengths of both types of diagrams, hybrid diagrams have been used (Richardson, 1986; Sterman, 2000); we and others call them system structure diagrams (SSD). They contain stocks and flows, feedback loops, intermediate variables, and delays, but they spare all the computational details which are not essential in order to represent the model structure graphically. An SSD of the LV model is provided in Figure 4.

With this much established, we now recapitulate the structural components used in system dynamics in addition to the dynamic systems representation:

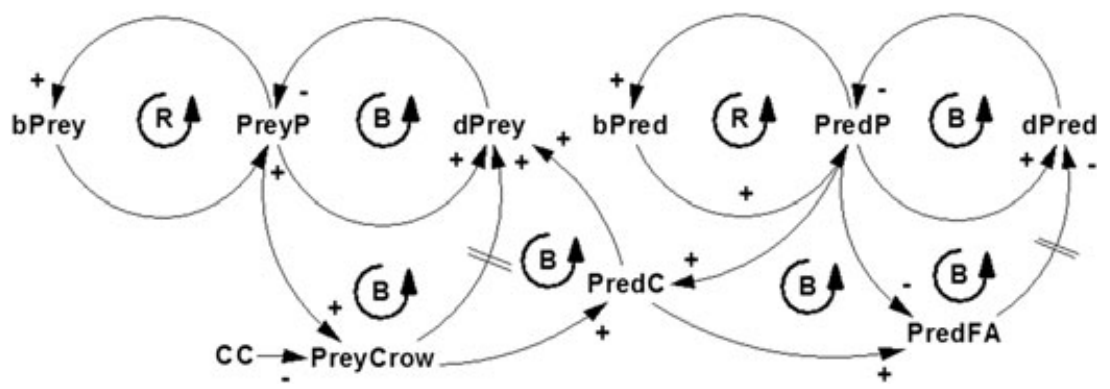

Figure 3. Causal loop diagram of the LV model 


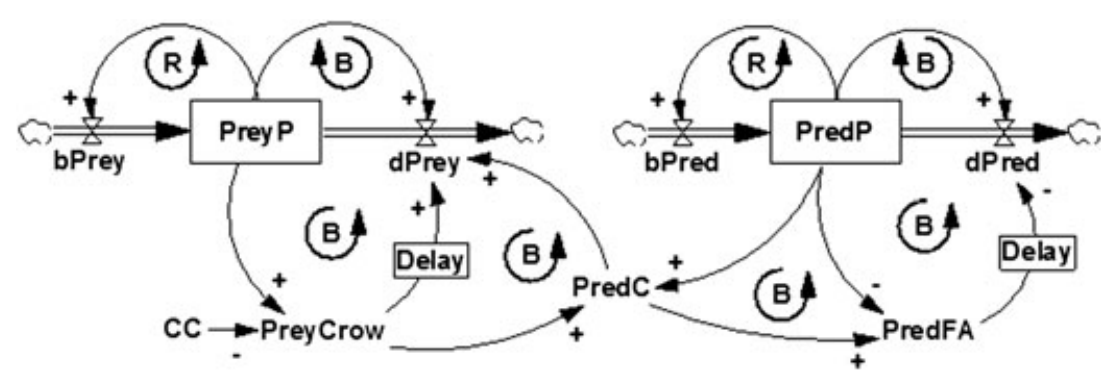

Figure 4. System structure diagram of the LV model

- Auxiliary variable/intermediate variable. These variables are neither stocks nor flows. Since only flows can influence stocks (Forrester, 1968b), auxiliary variables can be used only in the formulation of flows, e.g. crowdedness of prey, PreyCrow. Intermediate variables are often beneficial in the conceptualization and formulation of causal relationships because they provide clarity for human modelers and a non-technical audience. Intermediate variables could be avoided by collapsing them into rate variables which is, however, not appreciated in system dynamics modeling (Richardson and Pugh, 1981; Richmond, 2001).

- Feedback loop and its polarity. A feedback loop is a closed chain of causal relations. This is indicated in differential equations when a variable, e.g. Prey $P_{t}$, depends on its previous value. Each such loop operates as an endogenous mechanism and has a characteristic behavior depending on its polarity: either it reinforces initial changes (positive polarity) or it dampens them (negative polarity).

After the introduction of the fundamental structures of dynamic systems and system dynamics, we are going to synthesize them in the next section. This results in the elements for the formulation of the conceptual structure of an MMDS. The succeeding section then develops our proposed conceptual structure of an MMDS which is then used to operationally enhance the existing definition of an MMDS. Finally, both the benefits and limitations of this new structure are discussed.

\section{Discussion}

Synthesis of system dynamics and dynamic systems

The components of dynamic systems and system dynamics have been fleshed out in the previous sections. They are provided on the left-hand side of Table 2. On the right-hand side of that table, the components are related to dynamic systems theory and system dynamics. Black and white fields indicate whether the component is an element of the approach (black) or not (white). We distinguish between explicit and implicit consideration of the components. A component is explicitly accounted for when it is easily accessible to a broad audience (e.g. by graphical and iconic representation); a component is implicitly accounted for when it is in principle possible to represent the component by that approach; however, this representation is hidden, e.g. when the component is in the formulation of equations and therefore is not easily accessible by a general audience. For 
Table 2. Similarity of the elements of dynamic systems and system dynamics

\begin{tabular}{|c|c|c|c|c|c|c|}
\hline \multirow{2}{*}{ Component } & \multicolumn{2}{|c|}{ Dynamic system } & \multicolumn{4}{|c|}{ System Dynamics } \\
\hline & Explicit & Implicit & CLD & SFD & SSD & Implicit \\
\hline \multicolumn{7}{|c|}{ State variable/stock variable/level variable } \\
\hline \multicolumn{7}{|c|}{ Transition function/flow/rate } \\
\hline \multicolumn{7}{|c|}{ Intermediate variable } \\
\hline \multicolumn{7}{|c|}{ Causal link and polarity (positive, negative) } \\
\hline \multicolumn{7}{|c|}{ Nonlinear relationship } \\
\hline \multicolumn{7}{|l|}{ Delay } \\
\hline \multicolumn{7}{|l|}{ Circularity/feedback loop } \\
\hline Feedback loop polarity & & & & & & \\
\hline
\end{tabular}

CLD, causal loop diagram; SFD, stock and flow diagram; black boxes, "an element of"; grey boxes, "often not an element of"; unshaded boxes, "not an element of".

system dynamics, we distinguish the methods of CLD, SFD, and SSD. In the case of the SFD, we consider in addition the practice of how an SFD is used for representation (black: an element of; versus grey: often not an element of).

Table 2 shows that all of the components, with the exception of intermediate variables in the case of dynamic systems, are used at least implicitly to formulate models in both approaches. As one can see, all the components of the dynamic systems theory can be matched by the components available to system dynamics. This is supported also by Richardson (1999). Building on this, we can see that in the case of dynamic systems theory most of the components (e.g. causal links, polarities, and delays) are used only implicitly and are recognizable only by a mathematically educated beholder. Hence only two of the eight components are to be depicted explicitly. This situation changes with the use of the methods of system dynamics: seven of eight components are depicted by an SFD; stocks and flows, intermediate variables, and causal links and their polarities (black boxes) are clearly differentiated; delays, feedback loops, and feedback loop polarities are not always clearly represented (grey boxes), especially if the underlying model is larger (e.g. Sterman et al., 1997, 2007). A CLD is able to represent five of the eight components. The SSD is the most potent way to represent dynamic systems (Table 2). Such diagrams have been used more frequently in the last few years (e.g. Richardson, 1986; Sterman, 2000; Rudolph and Repenning, 2002; Schwaninger and Groesser, 2008; Perlow and Repenning, 2009). In the following, we address the individual components in more detail.

\section{Stocks and flows}

The traditional representation of dynamic systems uses state variables and transition functions. System dynamics uses the same components, which are called state and flow variables. There is a close correspondence between state and stock variables as well as flows and transition functions. This has already been shown by Richardson (1999).

Auxiliary/intermediate variables

Auxiliary variables are extensively used in system dynamics to explicate the causal structure of flow variables (Forrester, 1968b; Richardson and Pugh, 1981; Sterman, 2000; 
Lane, 2008). Forrester's (1968a) market growth model, for instance, has ten levels, 11 rates, and 27 intermediate variables (constants are not considered as intermediate variables); Homer's (1985) burnout model has six levels, six rates, and 18 intermediate variables; Forrester's (1969) urban dynamic model has 20 levels, 43 flows, and 140 intermediate variables. The LV model (Figures 1-3) has two levels, four flows, and three auxiliaries. Based on these examples, we can roughly state that the number of intermediate variables in a system dynamics model is normally at least as large as the sum of stock and flow variables. This finding is significant, since intermediate variables help to explicate the causal structure and therefore serve as means to improve transparency and comprehension. When intermediate variables would be aggregated into flow variables, the transparency of the system structure would be reduced. With the elimination of intermediate variables, the system dynamics representation converges towards a typical representation of dynamic systems theory. However, we emphasize the use of intermediate variables especially for the elicitation of mental models because we assume that humans, especially domain experts, do not have condensed and highly aggregated mental models, but rather use differentiations in the causal structure. These intermediary steps can best be captured by auxiliaries.

Causal relationships and polarities

Causal relationships are implied by the arithmetic operations in the equations. The representation in dynamic systems theory treats these causal relationships only implicitly; a layman observer cannot deduce them. The advantage of system dynamics is its explicit representation of causal relationships in all diagrams (CLD, SFD, SSD) because of the additional causal structure in the form of intermediate variables. In principle, the equations used in dynamic systems yield the same information in terms of causal links and polarities as a system dynamics representation, which, however, is only implicit.

Nonlinear relationships

Nonlinear relationships are only indirectly accessible in both approaches. In dynamic systems theory, they are embodied in multiplicative and divisional operations. The same holds in the case of system dynamics. In case of the absence of a more accurate mathematical relationship, system dynamics uses table or graph functions, i.e. bivariate relationships, to represent nonlinearities. These are normally engrained in intermediate variables. The notation of some system dynamics software packages tries to represent them explicitly (e.g. Powersim and Stella/iThink). Even with this software-specific notation, however, the largest amount of nonlinearities remains graphically inaccessible. In the commonly used standard system dynamics diagrams, i.e. CLD, SFD and SSD, nonlinearities are not represented.

Delays, feedback loops, and feedback loop polarity Dynamic systems theory does not directly reveal delays, feedback loops, and their polarity. Implicitly, however, they exist in forms of integrations and circular causality, and are therefore similar to the method of capturing these components in system dynamics. Again, the access to these concepts for a non-technical audience is made much easier by the diagrams system dynamics uses. To represent significant delays, feedback loops, and feedback loop polarity, a CLD or SSD has advantages, since they explicitly address all 
three components in an aggregated form to improve comprehensibility; an SFD, on the other hand, rather masks delays in equations.

Our discussion has shown that system dynamics can indeed represent all of the components of a dynamic system. This gives rise to the major difference: the structures and insights of a system are much more transparent, accessible, and comprehensible when that system is depicted by means of system dynamics. At the same time, the model of a dynamic system built using system dynamics will always be fully compatible with the original model. With the following three attributes in hand-elicitation of the MMDS structure which has been used in published research to measure MMDS use of the robust body of knowledge from dynamic systems theory (sections on "Dynamic systems" and "Synthesis of system dynamics and dynamic systems", above), and explicit treatment of the representational capabilities of system dynamics (sections on "System dynamics representation" and "Synthesis of system dynamics and dynamic systems", above)—we are justifiably confident that we have the building blocks for the conceptual structure of an MMDS. In the next section, we develop a comprehensive but also parsimonious conceptual structure. Thereby, we show which parts of the structure have been addressed by existing studies and which require additional attention.

\section{Conceptual structure}

After we have obtained the components of dynamic systems, we can develop a conceptual structure for MMDS. The elements which have been shown to be essential for describing dynamic systems are also important for inclusion in MMDS. As already defined by Doyle and Ford, a mental model's structure is held to be analogous to what it represents; therefore it should have a conceptual structure that is analogous to the representational devices used to represent such systems. Figure 5 shows the components of the conceptual structure. That structure has a hierarchical and balanced organization. The feedback loop, the concept absolutely necessary for endogenously representing dynamic systems, is at the highest level. The elements that constitute a feedback loop are variables and links. This part of the conceptual structure has already been measured by previous MMDS studies (the grey rectangles indicate this; see also Figure 1). The polarity of a feedback loop, though it has not been accounted for, is, however, a crucial structural element of an MMDS. Each of the three factors (variables, causal links, and feedback loop polarity) needs to be further differentiated to account for the specifics of dynamic systems. Variables need

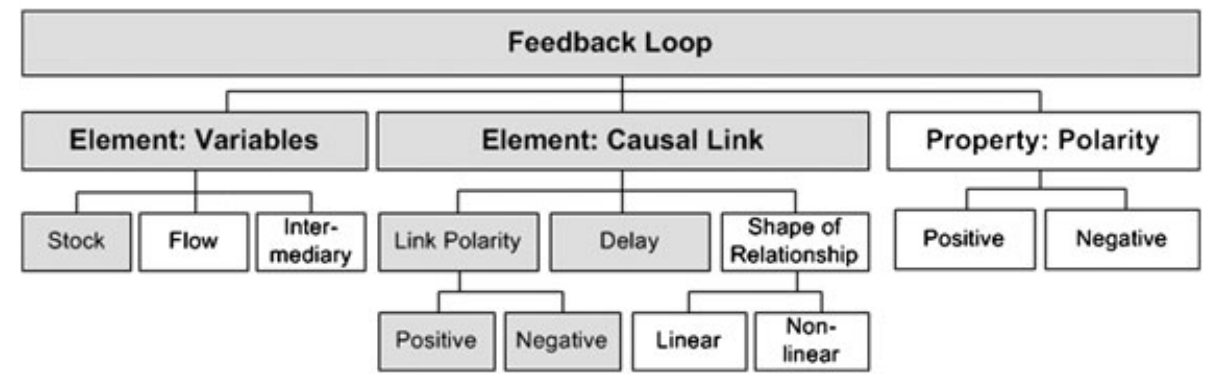

Figure 5. Conceptual structure of an MMDS. White areas indicate the new conceptual components which have not been accounted for by existing studies 
to be differentiated in stocks, flows, and intermediate variables; causal links contain link polarity (positive or negative), delays in the links, and the shape of relationships (linear or nonlinear); and the polarity of feedback loops can either be positive or negative. The white areas have not been covered explicitly by research into MMDS, even though they are highly relevant in accounting for the dynamics of such systems. The structure as laid out in Figure 5 can serve as a stepping stone for future research into the content of an MMDS.

The above section on "Research into mental models of dynamic systems" has shown that studies have measured additional properties, e.g. the length of a feedback loop ( Doyle et al., 2008; Verburgh, 1994) or the density of a mental model (Verburgh, 1994). These properties are covered by the conceptual structure in Figure 5 since the structure contains the basic elements these properties are composed of, e.g. links, polarities, and variables in the case of the measure "length of a feedback loop". We want to propose a conceptual structure that is parsimonious, but which also has the potential to account for specific operationalizations of measures. Hence we have collapsed some elements (e.g. length of a feedback loop) which are composed of the conceptual elements. In addition, we do not include the element of "link strength", which has been measured in the early studies of mental models (Langfield-Smith and Wirth, 1992). We exclude them from the conceptual structure since link strength is not a structural aspect of a link itself; it is a characteristic that is assigned to a link based on the interaction of the individual parts over time. Put differently, it is not possible to determine the strength of a link in advance without knowing other elements of the system. Moreover, the link strength (or link gain) is also subject to change over time.

In addition, we have excluded structural concepts such as "similarity between two mental models" (Markóczy and Goldberg, 1995; Schaffernicht and Groesser, 2011), since these are aspects that require more than one mental model. Considering this, we propose the conceptual structure of an MMDS (Figure 5). With these details of the structure of an MMDS, we can operationally elaborate the definition of an MMDS as provided by Doyle and Ford (1998, 1999):

A mental model of a dynamic system is a relatively enduring and accessible, but limited, internal conceptual representation of an external dynamic system (historical, existing, or projected). The internal representation is analogous to the external system and contains, on a conceptual level, reinforcing and balancing feedback loops that consist of causally linked stocks, flows, and intermediary variables. The causal links are either positive or negative, are either linear or non-linear, and can be delayed.

Figure 5 shows in addition which elements need more attention from researchers. Variables are differentiated in stock, flow, and intermediate variables. Initial studies have differentiated these variables (Sweeney and Sterman, 2000, 2007; Cronin and Gonzalez, 2007; Cronin et al., 2009), but their insights have not been connected to the discussion on MMDS. More explicit research is required to develop procedures and instruments by which these variables can be measured and distinguished. Moreover, a cause and its effect can be delayed. Schaffernicht and Groesser (2011) have developed an algorithm which accounts for the fact that links differ in the timeliness of their gains, but more research is required. Further research could include accounting for the shape of the relationships between two variables in MMDS. Ford and Sterman (1997) have developed a procedure to elicit nonlinear relationships from mental models; however, this approach has not yet 
been taken up in studies about MMDS. Moreover, the issue of polarity in frequently emerging feedback loops is also novel. Previous research has only accounted for the number of feedback loops a mental model might contain (Eden et al., 1992; Verburgh, 1994), but not for the polarities of the feedback loop. The conceptual structure in Figure 5 provides several possibilities for further research.

\section{Limitations}

Our contribution has several limitations. First, based on our work, the conceptual structure comprises components which have not been included before (Doyle and Ford, 1999). However, one might perceive that the conceptual structure could still be more detailed. Another possibility would be to account for the dominance of structures, i.e., for the temporal dominance of feedback loops (e.g., Ford, 1999; Kampmann and Oliva, 2006, 2009; Saleh et al., 2010). Another would be to account and detail behavior as a system unfolds over time (e.g. Ford, 1999; Saleh, 2002; Barlas and Bog, 2005). The aspects of structural dominance and system behavior are related to one another, since one of the principles of system dynamics is that the structure of the system determines the behavior of that system (Forrester, 1961; Richardson, 1995). But to account for the system behavior, a human being needs to deduce it mentally from the system structure and is therefore subject to severe biases, as many studies have shown (Dörner, 2006; Sweeney and Sterman, 2007; Cronin et al., 2009). We follow this reasoning and treat system behavior not as an element of the conceptual structure of an MMDS; rather, it is the result of the interaction of instances of this conceptual structure. Also the dominance perspective of the structure is a property which emerges over time and therefore builds on the conceptual structure but, is not a part of it.

The second limitation of our research is that we provide only a conceptual structurewe do not directly develop a measurement instrument. We have shown that some measures exist for some of the components, e.g. stock and flow differentiation (Sweeney and Sterman, 2000) or bivariate, nonlinear relationships (Ford and Sterman, 1997). We focus here on providing a set of components of an MMDS, not the instruments for measuring them. That will be a topic for a future study; Hall et al. (1994) provide a first review of existing elicitation methods. One issue which will emerge when the measurement instrument is developed is the differentiation in "revealed" versus "declared" conceptual structure-an important distinction which needs to be addressed. A further issue will be the representation of nonlinear relationships. Currently, such relationships are not explicitly represented (cf. Figure 4 and Table 2). One possibility of representing them explicitly would be to mark significant nonlinear relationships in CLDs and SSD; this would be similar to the practice of indicating significant delays between variables.

The third limitation of the paper is that we base the elicitation of components and the development of conceptual structure on existing-not original-evidence. We have decided to use three sources of existing research (research into MMDS, dynamic systems theory, and system dynamics) since we have perceived that a limited empirical elaboration of the conceptual structure would result in a structure with a lower level of validity. In addition, since dynamic systems theory is a mature and robust approach which describes dynamic phenomena sufficiently, we can have confidence that the conceptual structure is comprehensive. To validate this, we have used the conceptual structure as a coding scheme to analyze interview and workshop material which has elicited mental 
models. The result is that no additional category for the conceptual structure has emerged during our coding process. This initial application of the conceptual structure has increased our confidence that it covers the structural aspects which are required to describe dynamic systems. The next step is to use the conceptual structure in an empirical research project.

\section{Conclusion}

Humans perform only poorly in dynamic environments (Sweeney and Sterman, 2000, 2007; Dörner, 2006; Sterman, 2008; Cronin et al., 2009). One way to improve performance seems to be to understand their mental models of dynamic systems (MMDS). Until today, studies about MMDS have used intuitive and inconsistent operationalizations of the structural elements of such mental models. The definition of an MMDS (Doyle and Ford, 1999) does not specify the structural content of the internal conceptual representations. It is in this conceptual area that the paper's contribution can be positioned.

The result of the work in this paper is a conceptual structure of the content of an MMDS. In principle, a mental model of a dynamic system is composed of variables (stocks, flows, intermediate variables), causal links between the variables, link polarities, delays in the causal relationships, linear and nonlinear relations between variables, feedback loops, and the polarity of feedback loops. Our work provides a structure for these elements, and at the same time draws an explicit boundary around the elements which are required to represent dynamic systems. Existing studies about mental model research have accounted for only a part of this structure, which includes links, link polarities, and variables. Only a few studies have considered feedback loops as relevant elements. Most studies miss delays between cause and effect and the differentiation in stock, flow, and intermediate variables. It is not necessary to account for all the elements of the conceptual structure in one MMDS study, but it is nonetheless relevant to use the framework given by the conceptual structure to develop a commensurable body of research.

From a theoretical perspective, we have found that both dynamic systems theory and system dynamics manifest highly similar structural characteristics. By means of an analogy from dynamic systems theory, we demonstrate that one can develop a parsimonious conceptual structure for MMDS. Given this structure, we reason that, in their current state, MMDS-oriented studies run the risk of ignoring relevant aspects of dynamic systems because researchers focus too narrowly on variables and causal links. Based upon the proposed conceptual structure, researchers should be able to enhance the instruments they apply to mental model studies.

Our research can help in the comparison of mental models of dynamic systems. The application of our method can yield insights which can build on each other. A method for comparing MMDS, which measures our proposed structure of an MMDS has recently been published (Schaffernicht and Groesser, 2011). Research and better understanding of MMDS is of high relevance for improving real-world decision making.

To summarize the future research possibilities we have pointed to above, we see it chiefly in the development of instruments for empirical measurement of the elements of conceptual structures. Special attention will be needed for eliciting nonlinear relationships, delays, the differentiation between stocks, flows, and intermediate variables, feedback loops, and the polarity of feedback loops. Such attention is called for because the 
measured content of a mental model varies significantly with the measurement method used (Langan-Fox et al., 2001; Doyle et al., 2008). Any kind of comparative analysis between studies is futile unless the same measurement is employed.

\section{Acknowledgements}

We thank Andreas Größler and John Morecroft, Brian Dangerfield as the editor of System Dynamics Review, three reviewers of this journal as well as the participants of a discussion session at the International System Dynamics Conference 2010 in Seoul, Korea, for their helpful comments. In addition, we appreciate the financial support by the Swiss National Science Foundation (PBSGP1_133613) and by the internal project (E000402) of the University of Talca.

\section{Biographies}

Stefan N. Groesser is a professor of strategic management at the Bern University of Applied Sciences, Switzerland. In addition, he is a senior researcher for strategic management and system dynamics at the University of St Gallen, Switzerland, and a visiting scholar at the System Dynamics Group at MIT Sloan. He received degrees from the University of Stuttgart, Germany, in business administration and economics, and from the University of Bergen, Norway, in system dynamics. Stefan's research interests include strategic management, business models, mental models, and simulation methodology.

Martin Schaffernicht is Assistant Professor at the College of Business Administration of the Universidad de Talca, Chile. He holds a $\mathrm{PhD}$ in management science from the University of Montpellier II, France. His research interests are improvement and learning processes in organizations, mental models, and long-range planning.

\section{References}

Ackermann F, Eden C. 2011. Strategic management of stakeholders: theory and practice. Long Range Planning 44: 179-196.

Amaral LAN, Uzzi B. 2007. Complex systems: a new paradigm for the integrative study of management, physical, and technological systems. Management Science 53: 1033-1035.

Anderson P. 1999. Complexity theory and organization science. Organization Science 10: 216-232.

Argyris C. 1982. Reasoning, Learning, and Action: Individual and Organizational. Jossey-Bass: San Francisco, CA.

Arnold VI. 2006. Ordinary Differential Equations. Springer: Berlin.

Arrowsmith DK, Place C. 1990. An Introduction to Dynamical Systems. Cambridge University Press: Cambridge, UK.

Barlas Y. 1989. Multiple tests for validation of system dynamics type of simulation-models. European Journal of Operational Research 42: 59-87.

Barlas Y, Bog S. 2005. Automated dynamic pattern testing, parameter calibration and policy improvement. In Proceedings of the 23rd International Conference of the System Dynamics Society, Boston, MA.

Billard L. 1977. Lotka-Volterra predator prey models. Journal of Applied Probability 14: 375-381.

Brown C. 1995. Chaos and Catastrophe Theories: Nonlinear Modeling in the Social Sciences. Sage: Thousand Oaks, CA. 
Brunstein A, Gonzalez C, Kanter S. 2010. Effects of domain experience in the stock-flow failure. System Dynamics Review 26: 347-354.

Bryson JM, Ackermann F, Eden C, Finn CB. 2004. Visible Thinking: Unlocking Causal Mapping for Practical Business Results. Wiley: Chichester.

Capelo C, Dias JF. 2009. A system dynamics-based simulation experiment for testing mental model and performance effects of using the balanced scorecard. System Dynamics Review 25: 1-34.

Craik K. 1943. The Nature of Explanation. Cambridge University Press: Cambridge, UK.

Cronin MA, Gonzalez C. 2007. Understanding the building blocks of dynamic systems. System Dynamics Review 23: 1-17.

Cronin MA, Gonzalez C, Sterman JD. 2009. Why don't well-educated adults understand accumulation? A challenge to researchers, educators, and citizens. Organizational Behavior and Human Decision Processes 108: 116-130.

Desai M, Ormerod P. 1998. Richard Goodwin: a short appreciation. Economic Journal 108: 1431-1435.

Desthieux G, Joerin F, Lebreton M. 2010. Ulysse: a qualitative tool for eliciting mental models of complex systems. System Dynamics Review 26: 163-192.

Dörner D. 2006. Die Logik des Misslingens strategisches Denken in komplexen Situationen (5th edn). Rowohlt Taschenbuch Verlag: Reinbeck bei Hamburg.

Doyle JK. 1997. The cognitive psychology of systems thinking. System Dynamics Review 13: 253-265.

Doyle JK, Ford DN. 1998. Mental models concepts for system dynamics research. System Dynamics Review 14: 3-29.

Doyle JK, Ford DN. 1999. Mental models concepts revisited: some clarifications and a reply to Lane. System Dynamics Review 15: 411-415.

Doyle JK, Radzicki MJ, Trees WS. 1998. Measuring changes in mental models of dynamic systems: an exploratory study. In Proceedings of the 16th International Conference of the System Dynamics Society, System Dynamics Society, Quebec City, Canada.

Doyle JK, Ford DN, Radzicki MJ, Trees WS. 2002. Mental models of dynamic systems. In System Dynamics and Integrated Modeling: From the Encyclopedia of Life Support Systems, Barlas Y (ed.). EOLSS Publishers: Oxford.

Doyle JK, Radzicki MJ, Trees WS. 2008. Measuring change in mental models of complex dynamic systems. In Complex Decision Making: Theory and Practice, Qudrat-Ullah H, Spector MJ, Davidsen P (eds). Springer: Berlin; 269-294.

Eden C. 1988. Cognitive mapping. European Journal of Operational Research 36: 1-13.

Eden C. 1992. On the nature of cognitive maps. Journal of Management Studies 29: 261-265.

Eden C. 1994. Cognitive mapping and problem structuring for system dynamics model building. System Dynamics Review 10: 257-276.

Eden C, Ackermann F, Cropper S. 1992. The analysis of cause maps. Journal of Management Studies 29: 309-324.

Fokkinga B, Bleijenberg I, Vennix JAM. 2009. Group model building evaluation in single cases: a method to assess changes in mental models. In Proceedings of the 27th International Conference of the System Dynamics Society, Albuquerque, NM.

Ford DN. 1999. A behavioral approach to feedback loop dominance analysis. System Dynamics Review 15: 3-36.

Ford DN, McCormack DEM. 2000. Effects of time scale focus on system understanding in decision support systems. Simulation and Gaming 31(3): 309-330.

Ford DN, Sterman JD. 1997. Expert knowledge elicitation to improve formal and mental models. System Dynamics Review 14: 309-340.

Forrester JW. 1961. Industrial Dynamics. Productivity Press: Cambridge, MA. (Now available from Pegasus Communications, Waltham, MA) 
Forrester JW. 1968a. Market growth as influenced by capital investment. Industrial Management Review 9: 83-105.

Forrester JW. 1968b. Principles of Systems. MIT Press: Cambridge, MA. (Now available from Pegasus Communications, Waltham, MA)

Forrester JW. 1969. Urban Dynamics. Productivity Press: Cambridge, MA. (Now available from Pegasus Communications, Waltham, MA)

Fowler A. 2003. Systems modelling, simulation, and the dynamics of strategy. Journal of Business Research 56: 135-144.

Gary MS, Wood, RE. 2011. Mental models, decision rules, and performance heterogeneity. Strategic Management Journal 32: 560-594.

Gary MS, Kunc MH, Morecroft JDW, Rockard SF. 2008. System dynamics and strategy. System Dynamics Review 24: 407-429.

Goldvarg E, Johnson-Laird P. 2001. Naive causality: a mental model theory of causal meaning and reasoning. Cognitive Science 25: 565-610.

Goodwin RM. 1967. A growth cycle. In Socialism, Capitalism and Economic Growth: Essays Presented to Maurice Dobb, Feinstein CH (ed.). Cambridge University Press: Cambridge, UK; 54-58.

Hall RI, Aitchison PW, Kocay WL. 1994. Causal policy maps of managers: formal methods for elicitation and analysis. System Dynamics Review 10: 337-360.

Hodgkinson GP, Healey MP. 2008. Cognition in organizations. Annual Review of Psychology 59: $387-417$.

Homer J. 1985. Worker burnout: a dynamic model with implications for prevention and control. System Dynamics Review 1: 42-62.

Howick S, Ackermann F, Andersen D. 2006. Linking event thinking with structural thinking: methods to improve client value in projects. System Dynamics Review 22: 113-140.

Hufford MR, Witkiewitz K, Shields AL, Kodya S, Caruso JC. 2003. Relapse as a nonlinear dynamic system: application to patients with alcohol use disorders. Journal of Abnormal Psychology 112: 219-227.

Jensen E, Brehmer B. 2003. Understanding and control of a simple dynamic system. System Dynamics Review 19: 119-137.

Johnson-Laird PN. 1983. Mental Models Towards a Cognitive Science of Language, Inference, and Consciousness. Cambridge University Press: Cambridge, U.K.

Johnson-Laird PN. 1999. Deductive reasoning. Annual Review of Psychology 50: 109-135.

Johnson-Laird PN. 2001. Mental models and deduction. Trends in Cognitive Sciences 4: 434-442.

Kampmann CE, Oliva R. 2006. Loop eigenvalue elasticity analysis: three case studies. System Dynamics Review 22: 141-162.

Kampmann CE, Oliva R. 2009. System dynamics: analytical methods for structural dominance analysis. In Encyclopedia of Complexity and Systems Science. Springer: Berlin; 8948-8967.

Kozyk VV, Sydorov YI, Skvortsov IB, Tarasovska OB. 2010. Application of Lotka-Volterra model for description of duopolistic-duopsonic competition. Actual Problems of Economics 104: 252-260.

Kunc MH, Morecroft JDW. 2010. Managerial decision making and firm performance under a resource-based paradigm. Strategic Management Journal 31: 1164-1182.

Lane DC. 1999. Friendly amendment: a commentary on Doyle and Ford's proposed re-definition of "mental model". System Dynamics Review 15: 195.

Lane DC. 2008. The emergence and use of diagramming in system dynamics: a critical account. Systems Research and Behavioral Science 25: 3-23.

Langan-Fox J, Code S, Langfield-Smith K. 2000. Team mental models: techniques, methods, and analytic approaches. Human Factors 42: 242-271.

Langan-Fox J, Wirth A, Code S, Langfield-Smith K, Wirth A. 2001. Analyzing shared and team mental models. International Journal of Industrial Ergonomics 28: 99-112.

Langfield-Smith K, Wirth A. 1992. Measuring differences between cognitive maps. Journal of the Operational Research Society 43: 1135-1150. 
Lotka AJ. 1910. Contribution to the theory of periodic reaction. Journal of Physical Chemistry A 14: 271-274.

Maani KE, Cavana RY. 2007. Systems Thinking, System Dynamics: Managing Change and Complexity. Pearson Education: North Shore City, New Zealand.

Maani KE, Vandana V. 2001. Systemic thinking and complex problem solving: a theory building empirical study. In Proceedings of the 19th International Conference of the System Dynamics Society, Atlanta, GA.

Markóczy L, Goldberg J. 1995. A method for eliciting and comparing causal maps. Journal of Management 21: 305-333.

May RM, Oster GF. 1976. Bifurcations and dynamic complexity in simple ecological models. American Naturalist 110: 573.

McKelvey B. 1999. Avoiding complexity catastrophe in co-evolutionary pockets: strategies for rugged landscapes. Organization Science 1: 294-321.

Miller JH, Stadler PF. 1998. The dynamics of locally adaptive parties under spatial voting. Journal of Economic Dynamics and Control 23: 171-189.

Moxnes E. 2000. Not only the tragedy of the commons: misperceptions of feedback and policies for sustainable development. System Dynamics Review 16: 325-348.

Moxnes E. 2004. Misperceptions of basic dynamics: the case of renewable resource management. System Dynamics Review 20: 139-162.

Ogata K. 1998. System Dynamics. Prentice-Hall: Upper Saddle River, NJ.

Perlow LA, Repenning NP. 2009. The dynamics of silencing conflict. Research in Organizational Behavior 29: 195-223.

Plate R. 2010. Assessing individuals' understanding of nonlinear causal structures in complex systems. System Dynamics Review 26: 19-33.

Richardson GP. 1986. Problems with causal-loop diagrams. System Dynamics Review 2: 158-170.

Richardson GP. 1995. Loop polarity, loop dominance, and the concept of dominant polarity. System Dynamics Review 11: 67-88.

Richardson GP. 1999. Feedback Thought in Social Science and Systems Theory. Pegasus Communications: Waltham, MA.

Richardson GP, Pugh AL III. 1981. Introduction to System Dynamics Modeling with DYNAMO. Productivity Press: Cambridge, MA. (Now available from Pegasus Communications, Waltham, MA)

Richmond BM. 2001. An Introduction to Systems Thinking. High Performance Systems: Hanover, $\mathrm{NH}$.

Ritchie-Dunham J. 2002. Balanced scorecards, mental models, and organizational performance: a simulation experiment. $\mathrm{PhD}$ thesis, University of Texas at Austin.

Rosenhead J, Mingers J. 2001. Rational Analysis for a Problematic World Revisited: Problem Structuring Methods for Complexity, Uncertainty and Conflict (2nd edn). Wiley: Chichester.

Rudolph JW, Repenning NP. 2002. Disaster dynamics: understanding the role of quantity in organizational collapse. Administrative Science Quarterly 47: 1-30.

Saleh MM. 2002. The characterization of model behavior and its causal foundation. PhD thesis. University of Bergen, Norway.

Saleh M, Oliva R, Kampmann CE, Davidsen PI. 2010. A comprehensive analytical approach for policy analysis of system dynamics models. European Journal of Operational Research 203: 673-683.

Schaffernicht M. 2006. Detecting and monitoring change in models. System Dynamics Review 22: 73-88.

Schaffernicht M, Groesser SN. 2009. What's in a mental model of a dynamic system? On the conceptual structure and approaches to model comparison. In Proceedings of the 27th International Conference of the System Dynamics Society, Albuquerque, NM.

Schaffernicht M, Groesser SN. 2011. A comprehensive method for comparing mental models of dynamic systems. European Journal of Operational Research 210: 57-67. 
Schvaneveldt R. 1990. Pathfinder associative networks. In Studies in Knowledge Organization, Schvaneveldt R (ed.). Ablex: Norwood, NJ.

Schwaninger M, Groesser SN. 2008. Model-based theory-building with system dynamics. Systems Research and Behavioral Science 25: 447-465.

Seel NM. 2001. Epistemology, situated cognition, and mental models: "like a bridge over troubled water". Instructional Science 29: 403-427.

Senge PM. 1990. The Fifth Discipline: The Art and Practice of the Learning Organization. Currency Doubleday: New York.

Smith LB, Thelen E. 2003. Development as a dynamic system. Trends in Cognitive Sciences 7: 343-348.

Sterman JD. 1989a. Misperceptions of feedback in dynamic decision-making. Organizational Behavior and Human Decision Processes 43: 301-335.

Sterman JD. 1989b. Modeling managerial behavior: misperceptions of feedback in a dynamic decisionmaking experiment. Management Science 35: 321-339.

Sterman JD. 2000. Business Dynamics: Systems Thinking and Modeling for a Complex World. McGraw-Hill: Boston, MA.

Sterman JD. 2008. Risk communication on climate: mental models and mass balance. Science $\mathbf{3 2 2}$ : $532-533$.

Sterman JD. 2010. Does formal system dynamics training improve people's understanding of accumulation? System Dynamics Review 26: 316-334.

Sterman JD, Sweeney LB. 2002. Cloudy skies: assessing public understanding of global warming. System Dynamics Review 18: 207-240.

Sterman JD, Repenning NP, Kofman F. 1997. Unanticipated side effects of successful quality programs: exploring a paradox of organizational improvement. Management Science 43: 503-521.

Sterman JD, Henderson R, Beinhocker ED, Newman LI. 2007. Getting big too fast: strategic dynamics with increasing returns and bounded rationality. Management Science 53: 683-696.

Sweeney LB, Sterman JD. 2000. Bathtub dynamics: initial results of a systems thinking inventory. System Dynamics Review 16: 249-286.

Sweeney LB, Sterman JD. 2007. Thinking about systems: student and teacher conceptions of natural and social systems. System Dynamics Review 23: 285-312.

Verburgh L. 1994. Participative policy modeling applied to the health care insurance industry. PhD thesis, University of Nijmegen, Netherlands.

Volterra V. 1926. Variazioni e Fluttuazioni del Numero d'Individui in Specie Animali Conviventi. Memorie: Atti della Accademia Nazionale dei Lincei 2: 31-113.

Walsh JP. 1995. Managerial and organizational cognition: notes from a trip down memory lane. Organization Science 6: 280-321.

Warren K. 2008. Strategic Management Dynamics. Wiley: Chichester.

Wolfram S. 2002. A New Kind of Science. Wolfram Media: Champaign, IL. 